\title{
AN ANALYTICAL FORMULATION FOR THE LATERAL SUPPORT STIFFNESS OF A SPATIAL FLEXURE STRIP
}
M. Nijenhuis ${ }^{1}$,
J.P. Meijaard²,
J.L. Herder ${ }^{1}$,
S. Awtar ${ }^{3}$,
D.M. Brouwer ${ }^{1}$
1) Mechanical Automation and Mechatronics University of Twente
Enschede, The Netherlands
m.nijenhuis@utwente.nl

\begin{abstract}
This paper presents a framework for modeling the deformation and stiffness characteristics of static 3-D flexure strips (leaf springs), based on a discrete beam model that is suited for analytical calculations. As a case study, a closed-form parametric expression is derived for the lateral support stiffness of a parallel flexure mechanism.
\end{abstract}

\section{Continuous model}

A spatial Timoshenko beam with Reissner's finite strain measures - capturing shear, bending and torsion deformation - and linear elastic material behavior serves as a model for flexure strips.

\section{Discrete model}

A discretized version of the continuous model has been implemented in numeric flexible multibody software as a two-node beam element [1]. It is observed that a single such element captures stiffness characteristics of spatially deforming flexure strips with reasonable accuracy, owing to the inclusion of finite strain measures. As the mathematics of a single element remain comprehensible, the discrete model is well-suited for closed-form analysis. The available software implementation then serves as a calculation aid that facilitates the analytical modeling process.

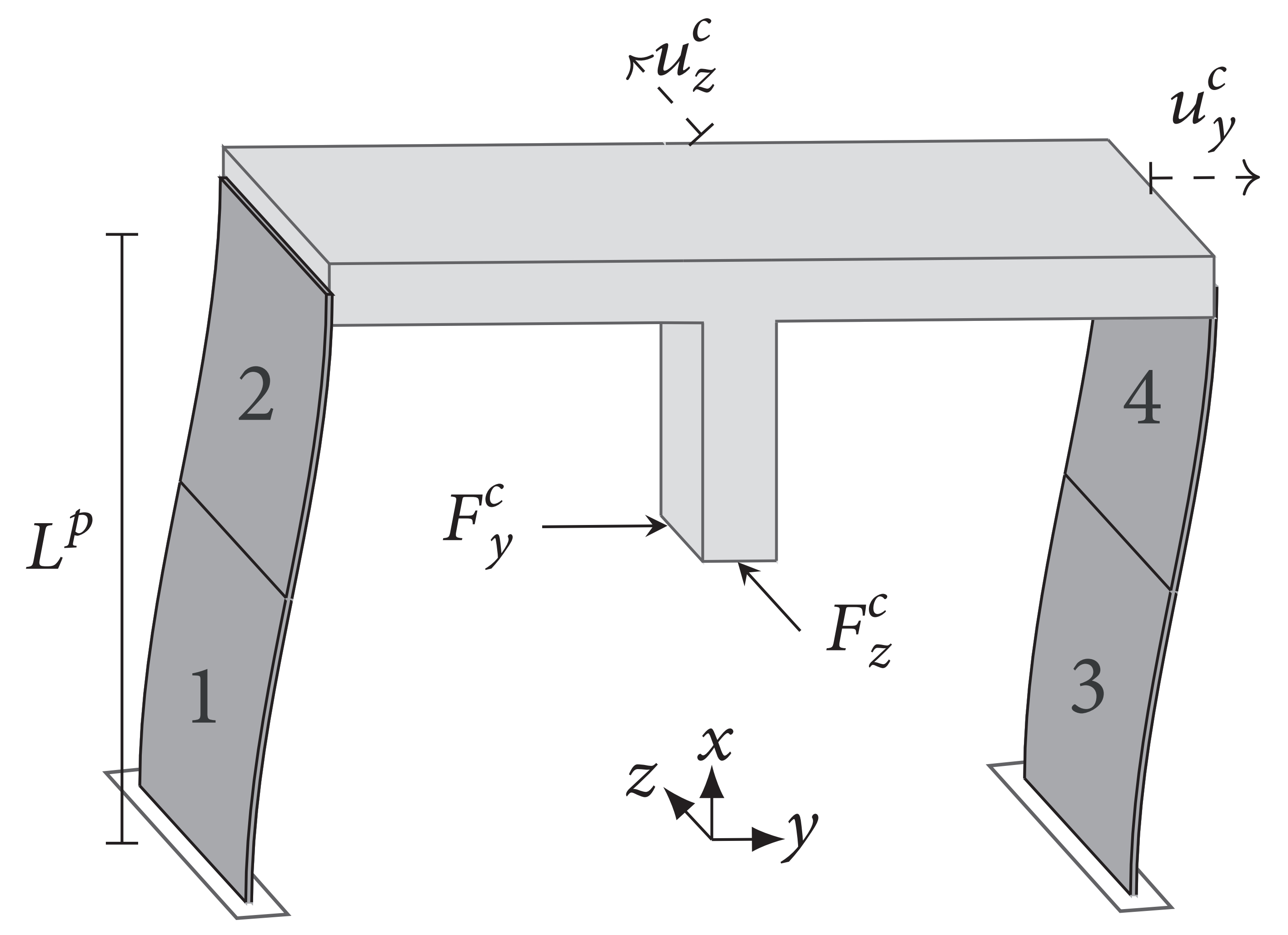

Figure 1: Parallel flexure mechanism modeled by four beam elements (1-4). Forces are applied in the center of compliance. The lateral support stiffness

(in z-direction) is investigated.

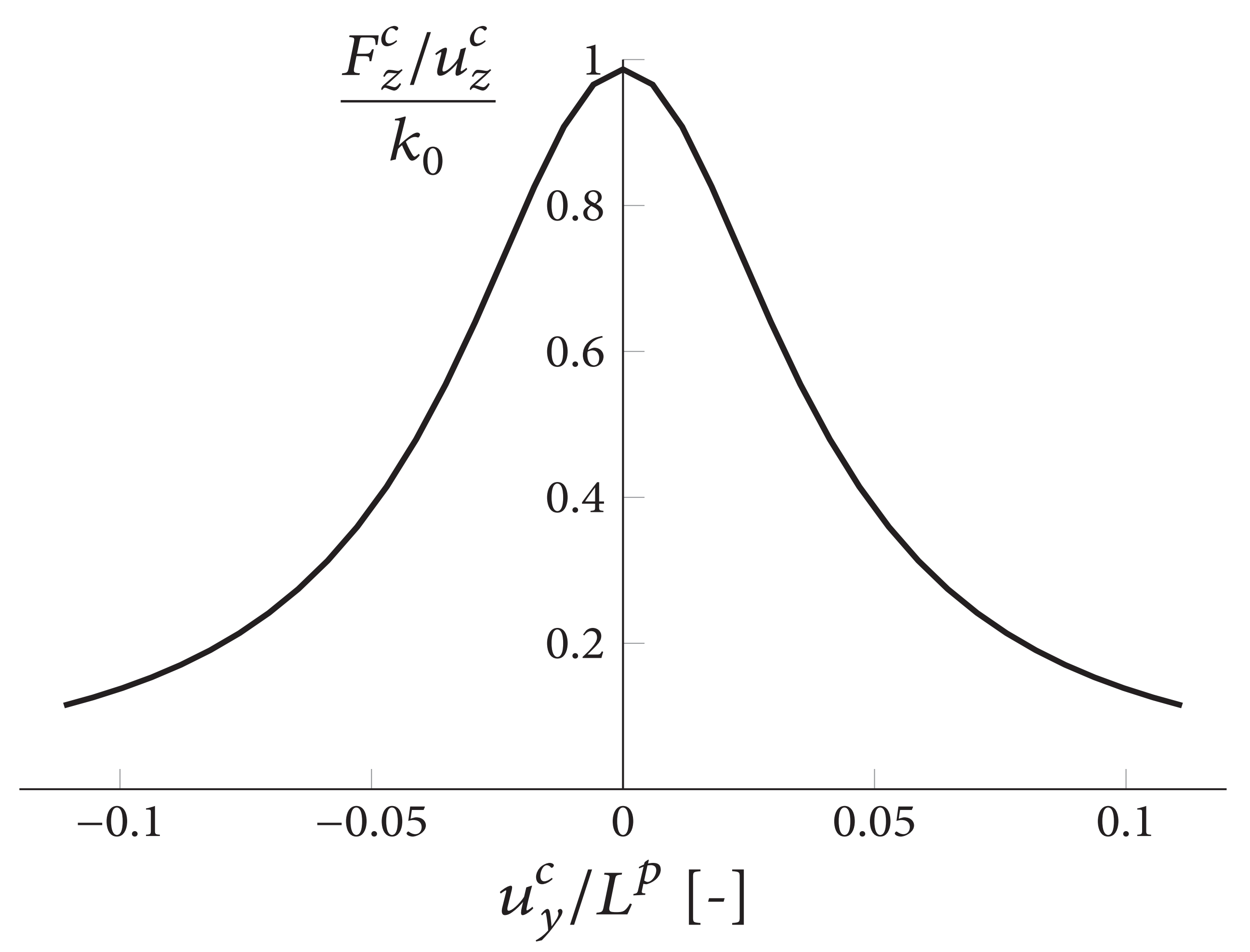

Figure 2: The (normalized) lateral support stiffness decreases significantly with (normalized) DOF displacement.

\section{Case study: \\ parallel flexure mechanism}

When a parallel flexure mechanism (figure 1) moves in the degree of freedom, the stiffness characteristics deteriorate: the lateral support stiffness decreases (figure 2). By using four discrete beam elements, a case-specific improvement of the torsion interpolation, and an approximation of the equilibrium configuration, this behavior is captured by the simple closed-form expression

$$
\frac{F_{z}^{c}}{u_{z}^{c}}=\left(\frac{\left(L^{p}\right)^{3}}{24 E I_{y}}+\frac{L^{p}}{2 k_{z} G A}+\frac{L^{p}}{56 G J}\left(u_{y}^{c}\right)^{2}\right)^{-1},
$$

where $F_{z}^{c} / u_{z}^{c}$ is the lateral support stiffness, $u_{y}^{c}$ the DOF displacement, and the other parameters have conventional definitions.

\section{Conclusion}

By incorporating a geometric non-linearity due to torsion and an effective torsional stiffness due to constrained cross-sectional warping, a compact parametric expression is obtained that gives insight into the lateral support stiffness of a parallel flexure mechanism. It is validated against FEA for parameters of practical interest.

\section{References}

[1] Jonker et al. Int. J. Nonlinear Mech. 2013, 53, 63. 